\title{
À la recherche des mécanismes de l'insulino-résistance
}

Oléagineux, Corps Gras, Lipides. Volume 10, Numéro 2, 115-8, Mars 2003, Approche biologique

Auteur(s) : Martine LAVILLE , Service d'Endocrinologie Diabétologie Nutrition, Hôpital E Herriot, Place d'arsonval, 69003 Lyon .

Author(s) : Martine LAVILLE

Résumé : Le concept d'insulino-résistance a surtout été développé depuis les années 1980, grâce à la description par $\mathrm{R}$ De Fronzo de la technique du clamp euglycémique hyperinsulinique, qui permet une quantification de la résistance à l'insuline. Il a ainsi été montré que l'insulino-résistance était une des caractéristiques du diabète non insulino-dépendant et de l'obésité. De nombreuses approches ont alors été développées pour mettre en évidence le siège de l'insulino-résistance. L'utilisation de traceurs du métabolismes du glucose et de la calorimétrie indirecte a permis de différencier, in vivo, le métabolisme hépatique du métabolisme périphérique et, l'oxydation du stockage. Le diabète de type 2 se caractérise ainsi par une augmentation de la production hépatique de glucose à jeun, par une insulino-résistance périphérique, par un défaut d'inhibition de la lipolyse et par des défauts des voies oxydative et non oxydative du glucose. Le caractère génétique du diabète de type 2 et de I'obésité a fait rechercher le \les gènes en cause dans l'insulino-résistance. De nombreuses mutations ont été mises en évidence dans les gènes impliqués dans l'action de l'insuline, mais cellesci n'expliquent actuellement que moins de $10 \%$ des diabètes non insulino-dépendant. Nous avons fait l'hypothèse que les altérations pouvaient porter non pas directement sur les gènes clés de l'action de l'insuline mais sur la régulation, notamment nutritionnelle, de l'expression de ces gènes. En effet, le diabète et l'obésité ont certes une composante génétique mais, ces pathologies ne se révèlent que dans un contexte nutritionnel favorable. Pour tester cette hypothèse, nous avons réalisé, chez des sujets sains, diabétiques de type 2 ou obèses des interventions nutritionnelles (restriction calorique, perfusion de lipides) ou hormonales (perfusion d'insuline) pour déterminer comment était modifiée l'expression des gènes et si des altérations étaient observées dans les états $d$ 'insulino-résistance. Les quantités d'ARNm de différents gènes cibles de l'action de l'insuline ont été mesurées par RT-PCR quantitative dans des biopsies de muscle et de tissu adipeux prélevées avant et après les différentes interventions. Nous avons ainsi pu montrer l'existence dans le diabète de type 2 d'un défaut spécifique de la régulation de la PI 3 kinase, de I'hexokinase II et de SREBP1c, alors que le défaut de régulation de Glut 4 est retrouvé dans divers états d'insulino-résistance. Nous étudions actuellement les mécanismes impliqués dans le défaut de régulation de ces gènes (étude des zones régulatrices des promoteurs et voie de signalisation impliquées dans la régulation). Nous recherchons également d'autres gènes dont la régulation pourrait être altérée en utilisant une approche plus large de DNA-chips. L'insulino-résistance n'a donc pas encore révélé tous ses secrets et promet encore de belles découvertes.

Summary : Insulin-resistance concept has been developed since the eighties after the description by De Fronzo of the euglycemic hyperinsulinemic clamp as a tool to quantify insulin-resistance. Insulinresistance has thus been established in obesity and insulin-resistance. The use of glucose tracer and indirect calorimetry has allowed distinguishing in vivo, hepatic and peripheral glucose metabolism, 
oxidation and storage. Type 2 diabetes is thus characterised by an increased hepatic glucose production, by a decreased insulin-stimulated glucose disposal rate by a defect in insulin lipolysis inhibition. Since obesity and type 2 diabetes are genetic disorders, defect in genes have been searched. Mutations in different genes have been found but it explains at least only $10 \%$ of type 2 diabetes. Our hypothesis has been that defects should not be on key-gene of insulin action, but on genes involved in nutritional regulation. To test this hypothesis we have realised in controls, type 2 diabetics and obeses, different nutritional (caloric restriction, lipid infusion..) or hormonal (insulin infusion) trials. In these trials we have determined the changes in key gene expression in muscle and adipose tissue biopsies obtained before and after the trials. Specific defects in the regulation of the expression of PI3 kinase, Hexokinase II and SREBP1c were observed in type 2 diabetes, whereas a defect in the regulation of Glut 4 expression was found in different insulin-resistant states. We are now looking for mechanisms involved in this defect of gene regulation (regulatory area of promotors, signalling pathways...). We are also in search of others genes which regulation could also been altered using the DNA-chips approach. The story of insulin-resistance is far from been closed.

Mots-clés : résistance à l'insuline, clamp euglycémique hyperinsulinique, obésité, diabète de type 2 , régulation de l'expression des gènes

Keywords : insulin-resistance, euglycemic hyperinsulinic clamp, obesity, type 2 diabetes, gene regulation

\section{ARTICLE}

\section{Auteur(s) : Martine LAVILLE}

Service d'Endocrinologie Diabétologie Nutrition, Hôpital E Herriot, Place d'arsonval, 69003 Lyon

Le développement dans les vingt dernières années de méthodes de mesure de la sensibilité à l'insuline a permis la mise en évidence d'une résistance à l'insuline dans de nombreux états pathologiques mais aussi physiologiques. Ceci a conduit au concept d'insulino-résistance qui a principalement pris son essor depuis la description par Andres et De Fronzo de la technique du clamp euglycémique hyperinsulinique [1]. Une insulino-résistance a ainsi été mise en évidence dans le cadre de l'obésité, du diabète non insulino-dépendant, du diabète lipoatrophique mais aussi de l'hypertension artérielle, des ovaires polykystiques, de la maladie de Cushing... Une insulinorésistance est aussi retrouvée en physiologie lors de la puberté, de la grossesse et du vieillissement. La démonstration de son association au risque cardio-vasculaire notamment dans le cadre du syndrome $X$ [2], fait de l'insulino-résistance un marqueur important qu'il convient de déterminer et justifie les nombreuses recherches passées, en cours et à venir dans ce domaine. ${ }^{1}$

${ }^{1}$ Texte de la conférence prononcée lors de la réception du prix Benjamin Delessert à Paris, en janvier 2003. 


\section{Caractérisation de la sensibilité à l'insuline}

\section{Définition}

La sensibilité à l'insuline est généralement caractérisée par l'action de l'insuline sur le métabolisme du glucose. En fait, l'action de l'insuline est complexe et intervient dans différents organes (principalement muscle, foie, tissu adipeux) et différents métabolismes (glucidique, lipidique, protéique) qui interagissent entre eux. L'insuline modifie l'activité d'enzymes clés mais elle joue également sur l'expression des gènes. L'insuline agit sur le métabolisme du glucose au niveau du foie, où elle diminue la production de glucose par inhibition de la néoglucogenèse et la glycogènolyse. Elle augmente la captation du glucose musculaire en translocant le transporteur du glucose Glut 4, elle stimule l'utilisation du glucose en augmentant la glycolyse et l'oxydation du glucose (par action sur la pyruvate déshydrogénase) ainsi que le stockage du glucose (par activation de la glycogène synthase). Elle agit sur le métabolisme lipidique en inhibant la lipolyse et en stimulant la lipogenèse à partir du glucose et de l'acétate. Elle agit sur le métabolisme protéique en stimulant les synthèses de protéines hépatiques et musculaires et surtout en réduisant leur catabolisme. Bien entendu, chacune de ces étapes pourra être siège de l'insulino-résistance.

\section{Mise en évidence}

Afin d'aller le plus loin possible dans la caractérisation de l'action de l'insuline, le clamp euglycémique hyperinsulinique peut être utilisé, associé à l'emploi de traceurs du métabolisme du glucose (ou/et des lipides et des protéines) et à la calorimétrie indirecte. On peut ainsi différencier in vivo le métabolisme hépatique du métabolisme périphérique, l'oxydation du stockage. La perfusion d'une dose élevée d'insuline permet la détermination de l'action maximale de l'insuline, alors que la réalisation d'une dose réponse à l'insuline, par l'emploi de perfusion successive de doses croissantes d'insuline permet de déterminer le k0.5 de l'insuline, soit l'insulinémie permettant d'avoir la moitié de l'action maximale de l'insuline. On voit ainsi que la sensibilité à l'insuline de la lipolyse est plus grande que celle de la production de glucose, elle même plus sensible que l'utilisation du glucose. Une insulino-résistance se traduit donc par un déplacement de la courbe vers la droite qu'il s'agisse de la courbe d'utilisation du glucose, d'inhibition de la production hépatique de glucose ou de la lipolyse. Ceci est caricatural dans certaines pathologies rares comme le diabète lipoatrophique dans lequel il existe une insulino-résistance périphérique majeure et une résistance extrême de la lipolyse [3].

On a bien sûr recherché des techniques simples pour caractériser la sensibilité à l'insuline, la réalisation de dose réponse à l'insuline restant lourde et réservée à des protocoles de recherche [4]. Le plus souvent l'insulino-sensibilité est mesurée avec une seule dose d'insuline suffisant pour bloquer la production hépatique de glucose et n'explorant donc que la sensibilité des organes périphériques. Des index simples ont été établis, il semble que le Quicki soit un des plus fiables: Quicki $=1 / \log (G)+\log (I)+\log F F A$ (où $G$ : glycémie à jeun $\mathrm{mmol} / \mathrm{l}, \mathrm{I}$ : insulinémie à jeun $\mathrm{pmol} / \mathrm{l}$; FFA acides gras libres à jeun en $\mathrm{mmol} / \mathrm{l}$ ) s'il est utilisé dans des conditions métaboliques stables [5].

\section{À la recherche des mécanismes de l'insulino-résistance}

C'est dans le diabète non insulino-dépendant et dans l'obésité que cette recherche a été la plus importante compte tenu des enjeux de santé publique de ces deux maladies et du fait que leurs mécanismes physiopathologiques ne sont pas encore parfaitement élucidés. Dans le diabète non 
insulino-dépendant, la résistance à l'insuline est marquée, avec une diminution de l'utilisation du glucose en relation avec un défaut de l'oxydation et du stockage du glucose. La production hépatique de glucose est augmentée le matin à jeun, mais modérément [6] et il existe un défaut d'inhibition de la production de glucose par l'insuline, bien que celle-ci puisse toujours être supprimée. L'inhibition de la lipolyse est également altérée. On sait que l'obésité et le diabète sont des maladies génétiques mais qu'elles ne se développent que face à un environnement " favorable ", en particulier en ce qui concerne la nutrition et l'activité physique. Le caractère génétique de ces affections a naturellement conduit, à la recherche de mutations dans les gènes clés de l'action de l'insuline. De nombreuses mutations ont ainsi été mises en évidence au niveau du récepteur à l'insuline, d'IRS1, de la glucokinase, de la glycogène synthase mais l'ensemble de ces mutations n'expliquent qu'une minorité des formes de diabète non insulino-dépendant et d'insulino-résistance. II a également été mis en évidence des mutations responsables de diabètes dans des facteurs de transcription comme HNF 1 et 4 et PPAR gamma. Pour aller plus loin, la recherche génétique dépasse actuellement la stratégie du gène candidat pour s'orienter vers un screening systématique. Dans ce contexte, nous avons émis l'hypothèse, que les anomalies retrouvées dans l'insulinorésistance qui ont un déterminisme génétique mais conditionné par des facteurs d'environnement, pourraient porter non directement sur les gènes cibles de l'action de l'insuline, mais sur des gènes responsables de la régulation nutritionnelle de l'expression de ces gènes clés. Pour ce faire nous avons cherché à étudier, in vivo chez l'homme, la régulation nutritionnelle et hormonales de gènes clés de l'action de l'insuline dans leur tissus cibles. La mise en route de ce projet nécessitait différentes mises au point :

1 : La réalisation de façon non agressive, de biopsies de muscle et de tissu adipeux, chez l'homme : II s'agit de biopsies réalisées sous anesthésie locale à l'aiguille pour le tissu adipeux sous cutané périombilical, à la pince de Weil-Blakesley pour le tissu musculaire pratiquées au niveau du faisceau quadricipital du muscle vaste-externe. Ces biopsies sont bien évidemment de petite taille : 60 à $80 \mathrm{mg}$ pour le muscle et 100 à $300 \mathrm{mg}$ pour le tissu adipeux.

2 : La mesure dans ces petits échantillons de quantité d'ARNm : ce qui nécessite la mise au point de techniques sensibles reproductibles et quantitatives d'ARNm. Ces techniques ont été mises au point dans le laboratoire [7]. Actuellement plus de 100 gènes peuvent ainsi être dosés.

3 : La réalisation des interventions hormonales et nutritionnelles. Celles-ci ont pour but de réaliser des modifications dans l'expression des gènes clés de l'action de l'insuline, de comparer ces modifications avec celles observées dans des groupes témoins. Ces tests dynamiques permettent de se libérer de modifications adaptationnelles de l'expression des gènes qui auraient pu être constatées à l'état basal. Les principales interventions réalisées ont été : la perfusion d'insuline en clamp euglycémique hyperinsulinique, la restriction calorique, la perfusion de lipides. Les sujets étudiés ont été des sujets obèses, des diabétiques non insulino-dépendants (DNID), des sujets normaux jeunes et plus âgés, des diabétiques insulino-dépendants comparables en HBA1c au diabétiques non insulino-dépendants (tableau 1). 
Tableau 1. Caractéristiques des sujets étudiés

\begin{tabular}{|l|l|l|l|}
\hline Sujets & Âge & IMC & Hba1c (\%) \\
\hline Témoins & $51 \pm 2$ & $23 \pm 1$ & $5.1 \pm 0.1$ \\
\hline Diabétiques de type 2 & $54 \pm 3$ & $0 \pm 1$ & $10.9 \pm 0.3$ \\
\hline Obèses non diabétiques & $43 \pm 4$ & $35 \pm 1$ & $5.5 \pm 0$ \\
\hline Diabétiques de type 1 & $33 \pm 3$ & $24 \pm 1$ & $9.2 \pm 0.3$ \\
\hline
\end{tabular}

Nous avons ainsi pu montrer que la perfusion d'insuline ( $1 \mathrm{mU} / \mathrm{kg}$.min pendant 3 heures) en clamp euglycémique hyperinsulinique était capable d'augmenter l'expression de gènes Glut 4, de la Rad, de la Phosphatidyl inositol-3-kinase, avec une diminution de l'expression de l'IRS1 et de la LPL dans le muscle [8]. Lors de la perfusion d'insuline, des défauts spécifiques de la régulation de l'expression des gènes ont été trouvés dans le DNID mais ni dans l'obésité ni dans le diabète de type 1. Les gènes porteurs de défaut de régulation sont l'hexokinase II, la P85 de la phosphatidyl-inositol-3 kinase, et SREBP1c dans le muscle. Ces anomalies ne sont pas corrigées par la normalisation glycémique au cours d'un traitement intensif. Dans le tissu adipeux, un défaut de régulation de SREBP1c par l'insuline est retrouvé dans le DNID et l'obésité $[9,10]$. Des défauts similaires ont été mis en évidence dans d'autres situations nutritionnelles telles que la restriction calorique par des Very Low Calory Diet (VLCD). Des sujets témoins, des sujets obèses et des DNID ont été soumis à 5 jours de diète à $250 \mathrm{Kcal}$. II a été observé chez les sujets témoins comme chez les sujets obèses, l'apparition d'une insulino-résistance alors que les DNID n'ont pas amélioré leur sensibilité à l'insuline. Ce déficit calorique a induit une adaptation génique avec une augmentation de l'expression du récepteur à I'insuline, de la Rad, de la PI3 kinase, d'UCP2 et d'UCP3. Cependant chez les diabétiques de type 2, alors que les autres gènes étaient induits comme chez les sujets contrôles et les obèses, il n'a pas été montré d'adaptation de la PI 3 kinase ni D'UCP 2 et $3[11,12,13]$ traduisant bien l'existence de défauts spécifiques de la régulation nutritionnelle de l'expression des gènes chez ces sujets.

Ainsi des manipulations différentes ont pu mettre en évidence des défauts de régulation de certains gènes qui semblent spécifique au DNID, comme l'Hexokinase II, la PI-3 kinase, SREBP1c. Ces défauts se retrouvent à la fois dans le muscle et le tissu adipeux, alors que d'autres défauts comme celui du Glut 4, retrouvés dans les syndromes d'ovaires polykystiques et dans les retards de croissance intrautérin, semblent plus spécifiques de l'insulino-résistance [14].

Pour aller plus loin et savoir si on peut considérer ces anomalies comme primitives, nous avons étudié la régulation par l'insuline de ces gènes chez des sujets sains apparentés au premier à des diabétiques de type 2 . Six des 20 sujets étudiés présentent effectivement les défauts observés chez les diabétiques de type 2 , en l'absence de toute insulino-résistance, argumentant en effet pour le caractère primitif de ces anomalies (données non montrées). 
Les hypothèses permettant de relier ces défauts de la régulation de ces gènes notamment par l'insuline sont multiples:

1 : Il peut s'agir d'une anomalie du signaling de l'expression des gènes par l'insuline (rôle de la PKB, de la p70 S6 kinase).

2 : Il peut s'agir de séquences consensus dans les promoteurs de gènes déréglés sur lesquelles viendraient se fixer des éléments communs pouvant être eux-mêmes responsables de la pathologie. 3 : Il y aurait d'autres gènes dont la régulation seraient altérée, mais qui n'auraient pas été mis en évidence avec notre approche.

Pour poursuivre ces hypothèses, des travaux se déroulent in vitro sur le modèle de myotubes en culture afin d'étudier les voies de signalisation de l'insuline et également d'étudier les promoteurs de I'Hexokinase II, de la PI-3 kinase et des différentes formes de SREBP. Par ailleurs, des approches plus large de pangénomique utilisant des puces à $A D N$ recherchent d'autres gènes ou familles de gènes dont la réponse pourrait être altérée dans le DNID ou d'autres états d'insulino-résistance. Nous présentons les premières données de la réponse à l'insuline du muscle de sujets normaux avec des puces de 30000 gènes.

Dans le futur, de nouvelles cibles vont nous conduire progressivement à mieux appréhender les multiples facettes de cette maladie polygénique si commune qu'est le DNID. Cette meilleure compréhension devrait faire naître de nouveaux espoirs thérapeutiques.

Il est évident que l'ensemble de ces travaux est le fruit d'une équipe et de collaborations intenses. Le noyau dur a associé des médecins, des chercheurs, des infirmières et des techniciennes qui se passionnent pour la recherche clinique et sans qui rien n'aurait été possible. Les liens avec les chercheurs de l'unité INSERM U 449 (ex 149) ont été déterminants et ont permis au clinicien que j'étais de se poser des questions et de savoir défendre les réponses que je trouvais. Arriver à échanger sur la " même longueur d'onde " sur un sujet de recherche avec des personnes d'horizon divers est certainement une des plus intenses jouissances intellectuelles. La recherche est un milieu passionnant fait de rencontres qui enrichissent. L'AFERO que j'ai l'honneur de présider fait partie de ce ces endroits. Je souhaite à tous ceux qui " entreront " un jour en recherche d'avoir la chance de faire de telles rencontres. C'est donc avec une émotion extrême que je reçois le prix Benjamin Delessert dont j'espère être digne.

\section{RÉFÉRENCES}

1. De Fronzo RA, Tobin J, Andres R. Glucose clamp technique : a method for quantifying insulin secretion and resistance. Am J Physiol $1979 ; 237$ : E214-23.

2. Reaven JM. Role of insulin resistance in human disease. Diabetes $1988 ; 37: 1595-607$.

3. Beylot M, Sautot G, Laville M, Cohen R. Metabolic studies in lipoatrophic diabetes : mechanism of hyperglycemia and evidence of resistance to insulin of lipid metabolism. Diabetes Metab, 1988 ; 14 : 20-4. 
4. Laville M, Andreelli F, Ducluzeau PH, Peyrat J, Riou JP. Evaluation de l'insulino-résistance en pratique clinique. Act Med Int-(Métabolismes-Hormones- Nutrition) 1998 ; $3: 1$-3

5. Bastard JP, Van V, Maachi M, Rabasa-Lhoret R, Jardel C, Bruckert E, Laville M, Hainque B. Incorporation of non esterified fatty acids into Quicki is not relevant in obese during diet inducing weight loss. Diabetes Metab $2002 ; 28$ : 333-4.

6. Rigalleau V, Beylot M, Laville M, Guillot C, Deleris G, Aubertin J, Gin H. Measurement of postabsorptive glucose kinetics in non-insulin dependent diabetes patients : methdological aspects. Eur J Clin Invest 1996 ; 26 : 231-6.

7. Aubœuf $D$, Vidal $H$. The use of the reverse transcription-competitive polymerase chain reaction to investigate the in vivo regulation of gene expression in small tissue samples. Anal Biochem 1997 ; vol. $245, n^{\circ} 2: 141-8$.

8. Laville M, Aubeuf D, Khalfallah Y, Vega N, Riou JP. Acute regulation by insulin of phosphatidylinositol-3-kinase, Rad, Glut 4, and lipoprotein lipase mRBNA in human muscle. J Clin Invest $1996 ; 98: 43-4$.

9. Andreelli F, Laville M, Ducluzeau PHD, Vega N, Vallier P, Khalfallah Y, Riou JP, Vidal H. Defective regulation of phosphatidyl-inositol-3-kinase gene expression in skeletal muscle and adipose tissue of non insulino-dependant diabetes mellitus patients. Diabetologia $1999 ; 42$ : 358-64.

10. Ducluzeau PHD, Perretti N, Laville M, Andreelli F, Vega N, Riou JP, Vidal H. Regulation of insulin of gene expression in human skeletal muscle and adipose tissue. Diabetes $2001 ; 50: 1134-42$.

11. Andreelli F, Laville M, Vega N, Riou JP, Vidal H. Regulation of gene expression during severe caloric restriction, lack of induction of p85 alpha phospatidyl inositol 3-kinase mRNA in skeletal muscle of patients with type II diabetes mellitus. Diabetologia $2000 ; 43: 356-63$.

12. Langin D, Larrouy D, Barbe $P$, Millet L, Viguerie-Bascand, Andreelli F, Laville M, Vidal H. Uncoupling protein-2 (UCP2) and uncoupling protein 3 (UCP3) expression in adipose tissue and skeletal muscle in human. Ont J Ob 1999, supp 6 ; S64-7.

13. Vidal $H$, Langin $D$, Andreelli F, Millet L, Larrouy $D$, Laville $M$. Lack of skeletal muscle uncoupling protein2 and 3 mRNA induction during fasting in type 2 diabetic patients. Am J Physiol 1999, 277(5 Pt 1) : E830-7.

14. Jaquet $D$, Vidal H, Hankard R, Czernichow P, Levy-Marchal C. Impaired regulation of glucose transporter 4 gene expression in insulin resistance associated with in utero undernutrition. $\mathrm{J}$ Clin Endocrinol Metab 2001 ; vol. 86, $\mathrm{n}^{\circ} 7$ : 3266-71. 